\title{
Polymer Dynamics in Repton Model at Large Fields
}

\author{
Anatoly B. Kolomeisky \\ Department of Chemistry, Rice University, Houston, TX 77005-1892, USA \\ Andrzej Drzewiński \\ Czestochowa University of Technology, \\ Institute of Mathematics and Computer Science, \\ ul.Dabrowskiego 73 , \\ 42-200 Czestochowa, Poland \\ and \\ Institute of Low Temperature and Structure Research, \\ Polish Academy of Sciences, P.O. Box 1410, 50-950 Wroctaw 2, Poland
}

Polymer dynamics at large fields in Rubinstein-Duke repton model is investigated theoretically. Simple diagrammatic approach and analogy with asymmetric simple exclusion models are used to analyze the reptation dynamics of polymers. It is found that for polyelectrolytes the drift velocity decreases exponentially as a function of the external field with an exponent depending on polymer size and parity, while for polyampholytes the drift velocity is independent of polymer chain size. However, for polymers, consisting of charged and neutral blocks, the drift velocity approaches the constant limit which is determined by the size of the neutral block. The theoretical arguments are supported by extensive numerical calculations by means of densitymatrix renormalization group techniques. 


\section{INTRODUCTION}

Dynamics of polymers in a dense medium is important in many chemical, biological and industrial processes. Current theoretical understanding of these phenomena is based on de Gennes reptation idea $\stackrel{1}{\underline{1}}$ According to this idea, the polymer chain cannot move easily in directions normal to its length because of the many obstructions in the system, instead, the polymer molecule diffuses in a snake-like motion along its contour.

The simplest model of polymer dynamics in systems with obstacles, which incorporates de Gennes reptation mechanisms, is the so-called repton model. It is a lattice model and it was first proposed by Rubinstein ${ }^{2}$ and later adapted by Duke as a model for the gel electrophoresis of DNA. $\underline{\underline{3}}$ Electrophoresis is a method of size-separation of charged polymers using an electric field. $\stackrel{4}{*}$ Numerous efforts have been invested in obtaining exact steady-state solutions of the repton model,$\underline{5.67 .8 .9 .10 .11 .12}$ however, with limited success. Van Leeuwen and Kooiman $\frac{7.8 .9}{}$ made an important advance in the analysis of the model by applying periodic boundary conditions. For the repton model with open boundaries formally exact but implicit formulas for the diffusion coefficient and the drift velocity are known. $\cdot \frac{5}{}$ However, except for small chains, $\underline{\underline{5}}$ there are no exact solutions for the stationary state. The more successful approach to understand the polymer dynamics in the repton model has been utilized in Monte Carlo computer simulations studies,$\frac{3.13 .14}{}$ although the results for large polymer sizes $N$ and/or large external fields are difficult to obtain. Recently, a new numerical method of investigation of the repton model based on the density matrix renormalization group (DMRG) approach has been presented. $\stackrel{15.16}{ }$

In this paper we investigate the dynamics of polymers in the repton model in the limit of very large external fields. In the repton model, this limit is less physical because it ignores two features of real electrophoresis that are important at large fields (although not at low fields): 1) transmission of tension through the segments of the polymer; and 2) the appearance of hernias, i.e., chain branching and creation of loops. Despite these facts the repton model in the limit of large fields still captures many qualitative properties of the gel electrophoresis of biological macromolecules. Exact analytic and asymptotic behaviors for the drift velocities are found using simple diagrammatic approach and analogy with asymmetric simple exclusion processes $\underline{17.18}$ This paper is organized as follows: the repton model is introduced in Sec. II, while in Sec. III the DMRG method is described. In Sec. 
IV the polymer dynamics at large fields is investigated using diagrammatic approach and analogy with asymmetric simple exclusion processes. Sec. V collects a series of numerical results of reptation in a strong field, while Sec. VI summarizes all results and concludes our paper.

\section{REPTON MODEL}

The repton model is tailored to describe the gel electrophoresis of DNA, and the gel is thought of as a space divided into cells, so that each cell corresponds to a pore in the gel. The macromolecule is divided into $N$ segments of equal length, and each segment is replaced by its midpoint (see Fig 1a). These points are called reptons, and each repton can consist of many monomer units. The number of reptons that each cell may accommodate is unlimited and self-avoidance effects are neglected.

As shown in Fig. 1b, the polymer chain consisting of $N$ reptons is connected by $N-1$ bonds. Each element of the chain is permanently confined to its own track running in the $x$ direction (parallel to the applied electric field), where $x$ is a coordinate that takes on only the discrete values. The polymer connectivity requires that the coordinates of adjacent reptons differ by only 0 or \pm 1 . Neighboring reptons that have a common value of their $x$ coordinate represent a cluster of successive reptons occupying a common cell in the gel. The clusters indicate an excess of reptons in cells; a polymer moves by the diffusion of these extra reptons. An interior repton $i(2 \leq i \leq N-1)$ can move only if it is the end repton of a cluster, and then it is allowed to move only in the direction of that one of its two neighbors which is not part of the cluster. This is shown in Fig. 1b by the arrows, $\uparrow$ (up) or $\downarrow$ (down). An end repton $(i=1$ or $N)$ is always allowed to move. If it is not part of a cluster, it can only move in the direction of its neighbor. If the end repton is part of a cluster, it can move in either direction.

For every allowed move $\uparrow$ or $\downarrow$ of a repton there is associated the transition probability per unit time, $B$ or $B^{-1}$, respectively, and $B$ defined as $B=\exp (\varepsilon / 2)$, where $\varepsilon$ is the dimensionless constant electric field. Since for every bond connecting two reptons at sites $i$ and $i+1(i=1, \cdots, N-1)$ the difference $x_{i+1}-x_{i}$ can have any of three values 0 or \pm 1 , the repton chain has $3^{N-1}$ possible distinct configurations irrespective of its location as a whole in the $x$ direction. Let $y=1, \cdots, 3^{N-1}$ numerate these polymer configurations. 
There is an equivalent representation of the repton model that maps it onto a onedimensional asymmetric simple exclusion model with two types of particles. $\frac{17,18}{1}$ In this representation each link between adjacent reptons in our polymer chain corresponds to a site on the new lattice. We associate a positive ("+") particle, or a negative ("-) particle, or a hole (" 0 ") with each site of the new lattice depending on the slope $\left(x_{i+1}-x_{i}\right)$ of the link in the original repton chain. This mapping is illustrated in Fig. 2 for one particular configuration of the polymer.

The dynamical rules for the repton model are easily expressed in the language of the asymmetric exclusion model. In the bulk the system evolves according to the following rules:

$(+)_{i}(0)_{i+1} \rightarrow(0)_{i}(+)_{i+1}$ with rate $B$

$(0)_{i}(+)_{i+1} \rightarrow(+)_{i}(0)_{i+1}$ with rate $B^{-1}$;

$(0)_{i}(-)_{i+1} \rightarrow(-)_{i}(+)_{i+1}$ with rate $B$;

$(-)_{i}(0)_{i+1} \rightarrow(0)_{i}(-)_{i+1}$ with rate $B^{-1}$.

At the left end of the lattice $(i=1)$ :

$(0)_{1} \rightarrow(+)_{1}$ with rate $B$;

$(+)_{1} \rightarrow(0)_{1}$ with rate $B^{-1}$;

$(-)_{1} \rightarrow(0)_{1}$ with rate $B$;

$(0)_{1} \rightarrow(-)_{1}$ with rate $B^{-1}$.

At the right end of the lattice $(i=N-1)$ :

$(0)_{N-1} \rightarrow(-)_{N-1}$ with rate $B$;

$(-)_{N-1} \rightarrow(0)_{N-1}$ with rate $B^{-1}$;

$(+)_{N-1} \rightarrow(0)_{N-1}$ with rate $B$;

$(0)_{N-1} \rightarrow(+)_{N-1}$ with rate $B^{-1}$.

Note, that here the exchange of positive and negative particles is not allowed.

\section{DENSITY MATRIX RENORMALIZATION}

In order to determine the properties of the reptation models we have used the densitymatrix renormalization group (DMRG). This technique enables us to study some global characteristics of polymers as the drift velocity and local ones as average shapes. The method was introduced in 1992 by S. White as an efficient algorithm to deal with a quantum 
Hamiltonian for one dimensional spin systems. $\underline{\underline{19}}$

This iterative basis-truncation method allows us to approximate eigenvalues and eigenstates with very high accuracy and in a controlled way $\underline{\underline{22}}$ The method is not restricted to quantum systems only; it has also been successfully applied to a series of problems, ranging from two-dimensional classical systems ${ }^{20}$ to stochastic processes: $\frac{15,16.21}{2}$ Using the formal similarity between the Master equation and the one-dimensional Schrödinger equation, the method of DMRG can also be applied to the Master Equation for the reptating chains ${ }^{15.16}$.

The number of configurations in the Hilbert space of the polymer grows very fast with its number of bonds $L=N-1$ (as $\left.3^{L}\right)$. Therefore, it is prohibitively difficult to solve exactly chains longer than $L=12$. But it is reasonable to eliminate the least probable (in the density matrix sense) states and keep only the most important ones. Although from this stage our calculation is not exact anymore, we can obtain very efficient approach if the weights of the discarded states are very small.

Starting with a small system (e.g. $L=4$ in our case), for which $H$ can be diagonalized exactly, one adds iteratively couples of reptons until the allowed (in the computational sense) size of the effective matrices is reached. Then further addition of new reptons forces one to discard simultaneously the least important states to keep the size of the effective matrices fixed. This truncation is done through the construction of a reduced density matrix whose eigenstates provide the optimal basis set (see Refs $\frac{19.22}{}$ for details). The size of the effective matrix is then substantially smaller than the original dimensionality of the configurational space $(3 m)^{2} \ll 3^{L}$. Generally, the larger is $m$, the better accuracy is guaranteed. In the present case, we keep this parameter up to $m=81$ (the stronger the fields, the larger the $m$ is necessary).

It is worth stressing that for a reptating chain, one has to face with the non-hermitian matrix $H$ (which becomes hermitian for zero driving field only). Since we are studying the very strong-field case we have to apply the non-hermitian variant of the standard DMRG algorithm, ${ }^{21}$ where one has to distinguish between the right and left eigenvector belonging to the same eigenvalue. Since $H$ is a stochastic matrix, its lowest eigenvalue is equal to 0 and the corresponding left eigenvector is proportional to the unit vector. The right eigenvector gives the stationary probability distribution. Generally, the DMRG method works best when the eigenvalues of $H$ are well separated. For long chains and strong fields the spectrum of $H$ gets an accumulation of eigenvalues near the zero eigenvalue of the stationary state. This 
hampers the convergence of the method seriously. Usually, enlarging the basis $m$ improves the accuracy substantially. However, for the reptation problem it helps very little and limits the systems under study with respect to the length.

To construct the reduced density matrix from the lowest eigenstates one has to diagonalize the effective matrix $H$ at each DMRG step. Therefore, we used the so-called Arnoldi method, ${ }^{23}$ which is known to be particularly stable for non-hermitian problems.

\section{HIGH-FIELD POLYMER DYNAMICS}

\section{A. Diagrammatic Approach}

Consider a polyelectrolyte (PE) molecule, where all charges in the polymer are of equal sign and of equal value, in the limit of very large applied electric fields. The formal expression for the drift velocity $V$ of a chain of size $N$ is given by ${ }^{5}$

$$
V=N^{-1} \sum_{y}\left(B r_{y}-B^{-1} s_{y}\right) p_{y}
$$

where $r_{y}\left(s_{y}\right)$ is the total number of $\uparrow(\downarrow)$ arrows on all of the reptons of chain in configuration $y$ and $p_{y}$ is the probability to find the chain in the configuration $y$. This equation implies that every configuration contributes to the drift velocity. Except for the case of small $N$, no general closed expression for $V$ is known.$^{\underline{5}}$ At the limit of large fields $(\varepsilon \rightarrow \infty)$ we expect that only few configurations play a major role in the polymer chain motions.

To single out relevant configurations, let us present them diagrammatically as follows. Put each configuration in a box, and connect the boxes with allowed transitions between the configurations by arrows. The direction along an arrow corresponds to a more probable transition (with rate $B$ ), while the opposite direction means a less probable transition (with rate $B^{-1}$ ). The illustration of the diagrammatic representation for the polymer chain of size $N=3$ is given in Fig. 3 .

Diagrams for general $N$ are more complicated but have the features that are already present for the $N=3$ diagram. There are cycles in the middle of the picture (for example, $y=2 \rightarrow y=1 \rightarrow y=4 \rightarrow y=2$ ). Each cycle has a size $N$. There are configurations which are less probable at any field $\varepsilon \neq 0$, such as the configuration $y=3$. There are configurations which are more probable (configuration $y=7$ is an example). 
In the limit of high fields there is a special class of so-called trap configurations. We define a trap configuration as one in which the allowed moves of the reptons are all highly unfavorable ones (against the field). The trap configurations for small chains are shown in Fig. 4.

The number of trap configurations quickly increases with $N$. As we can see from Fig. 4, there is a one trap configuration for $N=3$, two trap configurations for $N=4$, and four trap configurations for $N=5$. For $\varepsilon \gg 1$ we would expect to find a polymer chain mainly in these trap configurations in the stationary state. But a closer look at our diagrammatic picture shows that only one (for odd $N$ ) or two (for even $N$ ) trap configurations will be real traps. These real traps have a symmetric "U" shape (consistent with experimental observations of large-field behavior of DNA molecules in gel electrophoresis ${ }^{24}$ ).

To demonstrate this we look at two possible trap configurations of an $N=5$ polymer chain (see Fig. 5). Suppose we find a polymer molecule in the configuration $y=2$ (see Fig. 5a). For $\varepsilon \rightarrow \infty$ there is a very small but nonzero probability for a transition from the trap configuration $y=2$ to the configuration $y=1$. Then with only probability $1 / 2$ the chain returns to the trap. Thus at longer times the probability to find the chain in this trap configuration decreases to zero, therefore implying that the chain escapes this trap. In contrast, if the chain is in the trap configuration $y=5$ (see Fig. 5b), and it undergoes a transition to the configuration $y=4$ or $y=6$, then with overwhelming probability the system returns back to the real trap configuration. This kind of argument can be extended to a chain of any size.

Using this idea we can calculate the probabilities of different configurations in the highfield limit. In the example of $N=5$ (Fig. $5 \mathrm{~b}$ ) the probability to find the trap configuration $y=5$ is $p_{5} \sim 1$. Then because of the local detailed balance $p_{4}=p_{6} \sim B^{-2}$. For general $N$ this trend will continue: each move up on the diagram away from the real trap decreases the probability of configurations by $B^{2}$. In this case the expression for the drift velocity becomes:

$$
V=N^{-1}\left[-p_{5}(2 / B)+p_{4}(B-3 / B)+p_{6}(B-3 / B)+\cdots\right)=N^{-1}\left(-6 / B^{3}+\cdots\right] .
$$

The structure of the equation implies that the contributions to the drift velocity from the trap configurations and from the configurations leading to the traps cancel each other. The process of cancellation continues as we go up on the diagram until the branching. By 
branching we call the existence of another probable way of moving the chain, not leading to the real trap configuration. For example, in Fig. $4 \mathrm{~b}$ the configuration $y=1$ is a place of branching because there are two escape routes from this configuration. By following back from the trap configuration on the diagram, we effectively move a zero-slope bond from the ends to the middle of the chain (see Figs 3 and $5 \mathrm{~b}$ ). Then for odd $N$ there are $(N-1) / 2$ steps from the trap configuration before the branching, while for even $N$ there are $(N-2) / 2$ steps. Thus the probability of the configuration which gives a non-zero contribution to the drift velocity is $\sim B^{1-N}$ (for odd $N$ ) or $\sim B^{2-N}$ (for even $N$ ). As a result, in the limit of large electric fields the drift velocity is given by

$$
\begin{aligned}
& V \sim B^{2-N}, \quad N-\text { odd } \\
& V \sim B^{3-N}, \quad N-\text { even }
\end{aligned}
$$

These expressions are valid for any $N$ and large fields (when $N E \gg 1$ ). However, we cannot determine explicitly the constants in front of field-dependent terms. We can only conjecture that it grows also exponentially with the polymer size. Exponential decrease in the drift velocity of polymers has been observed in recent computer simulations of gel electrophoresis with cage model $\stackrel{27}{2}$

The diagrammatic approach can also be used to treat polyampholytes (PA), the polymer molecules with charges of different sign. Specifically, consider alternating PA, where positive and negative charges along the polymer backbone alternate with each other. Then in the limit of large fields we expect only one "crown"-shaped trap configuration as a real trap.

To calculate the drift velocity, we again utilize Eq. (10), which is also valid for polyampholytes, but the meaning of the parameters $r_{y}$ and $s_{y}$ changes. Now for a given configuration $y, r_{y}$ is the difference between the number of $\uparrow$ moves (positive direction of the motion) with the probability $B$ minus the number of $\downarrow$ moves with the probability $B$. Similarly, $s_{y}$ is the difference between the number of $\downarrow$ moves with the probability $B^{-1}$ minus the number of $\uparrow$ moves with the probability $B^{-1}$. It is obvious that $r_{y}$ and $s_{y}$ can be negative, positive or zero, in contrast to the $\mathrm{PE}$ case where these parameters were always non-negative.

For even $N$ the drift velocity $V=0$ at all values of the applied electric field. This can be seem from the fact that for any configuration $y$ with parameters $r_{y}$ and $s_{y}$ there is a configuration $y^{*}$ with the parameters $-r_{y}$ and $-s_{y}$ related to $y$ by inversion. If the configuration $y^{*}$ and $y$ are the same then $r_{y}=s_{y}=0$. 
The case of odd $N$ is different. For $\varepsilon \rightarrow \infty$ the diagrammatic approach predicts that the drift velocity is asymptotically given by

$$
V \sim B^{-3}
$$

for all $N$. For the smallest alternating PA chain, $N=3$, we can calculate the drift velocity at fields directly by using transition-rate matrix method. $\frac{5}{5}$ It gives us

$$
V=\frac{4\left(B-B^{-1}\right)}{3\left(B^{4}+B^{-4}\right)+7\left(B^{2}+B^{-2}\right)+16},
$$

which in the limit of very large $\varepsilon$ reduces to Eq. (4).

\section{B. Analogy with Asymmetric Exclusion Processes}

Similarly to the case of polyelectrolytes and polyampholytes, the repton model can be applied to polymers with charged and neutral segments. Here we consider a chain comprised of two blocks. The left block consists of $k$ charged reptons (the charges of the same sign), and the right block consists of $N-k$ neutral reptons. The experimental realization of such system, for example, is gel electrophoresis of DNA-neutral protein complex. ${ }^{25}$

Using the mapping onto the one-dimensional asymmetric simple exclusion model as described in Sec. II we identify a negative bond variable $\left(j_{i} \equiv x_{i+1}-x_{i}=-1\right)$, a positive bond variable $\left(j_{i}=+1\right)$, and a zero bond variable $\left(j_{i}=0\right)$ with a $p$-particle, $m$-particle, and vacancy, respectively. In the steady-state the current of $p$-particles $J^{(p)}$ is the same at every site of the lattice but different from the current of $m$-particles $J^{(m)}$ as can be seen from the asymmetry of the system. We assume that the positive direction for the current of $p$-particles is from left to right, and the positive direction for the current of $m$-particles is from right to left. Then the drift velocity of the original repton model is connected to the steady-state currents in this asymmetric simple exclusion model by

$$
V=J^{(p)}+J^{(m)}
$$

It is convenient to introduce a difference of the currents $\Delta J$

$$
\Delta J=J^{(p)}-J^{(m)}
$$


The average densities $\left\langle p_{i}>\right.$ and $<m_{i}>$ determine the currents. At the left end of the polymer $(i=1)$ the currents are

$$
\begin{gathered}
J^{(p)}=B<1-p_{1}-m_{1}>-B^{-1}<p_{1}> \\
J^{(m)}=B<m_{1}>-B^{-1}<1-p_{1}-m_{1}>.
\end{gathered}
$$

For the sites $i=1, \cdots, k-1$ of the charged block we have

$$
\begin{gathered}
J^{(p)}=B<p_{i}\left(1-p_{i+1}-m_{i+1}\right)>-B^{-1}<\left(1-p_{i}-m_{i}\right) p_{i+1}> \\
J^{(m)}=B<\left(1-p_{i}-m_{i}\right) m_{i+1}>-B^{-1}<m_{i}\left(1-p_{i+1}-m_{i+1}\right)>.
\end{gathered}
$$

For the sites $i=k, \cdots, N-2$ of the neutral block we have

$$
\begin{gathered}
J^{(p)}=<p_{i}\left(1-p_{i+1}-m_{i+1}\right)>-<\left(1-p_{i}-m_{i}\right) p_{i+1}> \\
J^{(m)}=<\left(1-p_{i}-m_{i}\right) m_{i+1}>-<m_{i}\left(1-p_{i+1}-m_{i+1}\right)>.
\end{gathered}
$$

The currents at the right end of the lattice $(i=N-1)$ are given by

$$
\begin{gathered}
J^{(p)}=<p_{N-1}>-<1-p_{N-1}-m_{N-1}> \\
J^{(m)}=<1-p_{N-1}-m_{N-1}>-<m_{N-1}>.
\end{gathered}
$$

Introducing an average density of vacancies $<n_{i}>=<1-p_{i}-m_{i}>$ we obtain from the Eqs. (8)

$$
V=\frac{B-B^{-1}}{2}\left(1+<n_{1}>\right)-\frac{B+B^{-1}}{2}\left(<p_{1}>-<m_{1}>\right),
$$

and

$$
\Delta J=\frac{B+B^{-1}}{2}\left(3<n_{1}>-1\right)+\frac{B-B^{-1}}{2}\left(<p_{1}>-<m_{1}>\right) .
$$

From Eqs. (10 11] one can conclude that

$$
\begin{gathered}
\Delta J=<n_{i+1}>-<n_{i}>, \quad i=k, \cdots, N-2 \\
\Delta J=1-3<n_{N-1}>.
\end{gathered}
$$

Consequently,

$$
<n_{i}>=(3 N-3 i-2)<n_{N-1}>-(N-i-1), \quad i=k, \cdots, N-1 .
$$

It is impossible to find exact solutions of the system ([6] [15]) for general values of the field because the number of variables exceeds the number of equations, except in the limit $\varepsilon \rightarrow \infty$. 
In this limit one realizes that $<n_{1}>=<n_{2}>=<n_{3}>=\cdots=<n_{k}>=0$. Then from Eq. (15) one can obtain

$$
\begin{gathered}
<n_{N-1}>=\frac{N-k-1}{3(N-k)-2}, \\
\Delta J=\frac{1}{3(N-k)-2} .
\end{gathered}
$$

Now comparing Eqs. (13) and (12) in the limit of large applied field we obtain the following expression for the drift velocity

$$
V=\Delta J=\frac{1}{3(N-k)-2}
$$

For $k=1$ this system was investigated in $\operatorname{Ref}^{17}$. In that paper Monte Carlo simulations were used to extract the drift velocity at any value of the field. Also an exact solution in the infinite field-limit was found, which for $k=1$ reproduces Eq. (18).

Another way to check our results is to calculate directly the drift velocity for small chain $N=3$ by using transition-rate matrix method. $\frac{5}{}$ Then calculations for $k=1$ yield

$$
V=\frac{B^{2}-B^{-2}}{4\left(B^{2}+B^{-2}\right)+3\left(B+B^{-1}\right)+4},
$$

which in the limit $\varepsilon \gg 1$ approaches to $1 / 4$, in agreement with Eq. (18).

For $k=2$ and $N=3$ the drift velocity is given by

$$
V=\frac{B^{2}-B^{-2}}{\left(B^{2}+B^{-2}\right)+3\left(B+B^{-1}\right)+1} .
$$

In the limit $\varepsilon \rightarrow \infty$ we have $V \rightarrow 1$, again in agreement with our general expression (18).

\section{NUMERICAL RESULTS}

Since we are interested in behavior of polymers in a very strong electric field we have to analyze a strongly non-hermitian Hamiltonian. Although, from computational reasons, it limits our considerations to chains of a moderate length, studying them we can obtain a clear picture.

Note that the number of reptons is always odd in our DMRG calculations. This provides an additional constraint to our numerical method. 


\section{A. Polyelectrolytes}

In order to verify which configurations dominate for strong fields, we can calculate the average slopes $\left\langle y_{i}\right\rangle$ as function of the bond position along a chain. They are always symmetrical, which is a consequence of the intrinsic symmetry between a head and a tail for the uniformly charged chains. Summing elements $\left\langle y_{j}\right\rangle$ up to a certain repton the average shape of a polymer can be found. As a point of reference, the position of the first repton is established as a zero.

As shown in Fig.6, with the increasing field $\varepsilon$ the polymer configuration is changing from the horizontal to the U-shape form. In the left part of a chain the average slopes $\left\langle y_{i}\right\rangle$ are going to the value -1 , whereas in the right part they reach the value +1 . That is why the configuration minimum goes to the value of $(N-1) / 2$. At the same time, it means that when $\varepsilon$ grows, the real trap configuration is dominating more and more, in a perfect agreement with our expectation.

In Fig.7 we have collected the DMRG results for the drift velocity. They are calculated from the expression given by van Leeuwen and Kooiman. ${ }^{26}$ Its dependence on the electric

field (in the inset) is in agreement with previous results. 13 For small fields one can observe a linear dependence, which is followed by a maximum and an exponential decay for large fields. The presence of an exponential decay results from the dominating role of the trap configurations. Polymers are stuck on obstacles being pulled at both arms. Since the tension is not translated in the repton model, the resulting drift velocity of the polymers decreases strongly with the field.

In order to find out the decay exponent we have presented our results in the linear-log scale (Fig. 7). As one can see, in the large field limit the agreement with the expression found by the diagrammatic approach (Eq.(3)) is perfect for all calculated polymer chains.

\section{B. Polyampholytes}

DMRG method can also be extended to calculate properties of polyampholytes. As an example, we have studied alternating PA chains. Since only odd-number reptons polymer chains are considered the sign of end charges is always the same (positive in our case).

The average shapes for polymers of size $N=11$ are presented in Fig. 8. As expected, a 
polymer chain configuration is approaching the crown shape at large fields. Since in our plot the chain is relatively small, the influence of end reptons is still relatively large. Generally, when $\varepsilon$ grows, one can expect that for all $N$ the amplitude of the middle part goes to values of a range of a few bond lengths.

Our calculations for the drift velocity, in contrast to uniformly charged chains, indicate that the decay exponent does not depend on an alternating chain length (Fig. 9). This conclusion is in a full agreement with our theoretical predictions: see Eq.(44).

\section{Polymers Consisting of Neutral and Charged Blocks}

DMRG also allows us to investigate the polymer chains consisting of neutral and charged blocks. Let us first consider a chain consisting of all neutral reptons except one charged repton at the end. Hence, according to our notation from Sec.IV, $k=1$. Obviously, the average shape here differs substantially from the case where all reptons charged. Nevertheless, the resulting shapes are intuitively easy to understand. As one can see in Fig. 10, the charged repton at the left end pulls the whole chain, and this effect is stronger at larger fields.

In order to determine a size-dependence of drift velocity for different polymers with neutral and charged blocks, we have studied two cases: $k=1,2$. The results are presented in Figs. 11 and 12, and they indicate that the limit behavior at large fields is in a perfect agreement with Eq.(18). Note also that the absolute values of drift velocities are larger for the polymers with more charged reptons, i.e., the same-size polymer chains with $k=2$ move significantly faster than the polymers with only one charged end repton, in agreement with intuitive expectations. Our results agree also with Monte Carlo simulations ${ }^{17}$ and numerical calculations ${ }^{28}$ for a magnetophoresis, which corresponds to our $k=1$ case.

\section{SUMMARY AND CONCLUSIONS}

We have analyzed a lattice model of reptation (the Rubinstein-Duke model) to study the dynamics of polymer in a dense medium. Asymptotically exact results in the limit of large applied fields have been obtained by means of simple diagrammatic approach and using the analogy with asymmetric simple exclusion processes. Our theoretical arguments are based on a realistic assumption that at large fields only a few configurations are important for 
polymer dynamics.

The method was successfully used for different types of polymers. For polyelectrolytes and polyampholytes, we find that the drift velocity is exponentially decreasing as a function of an external field. For PE chains the exponent is a function of polymer size, while for PA it is independent of polymer size. For polymers consisting of neutral and charged blocks the situation is very different. In this case, at large fields the drift velocity approaches a constant value, which depends on the size of the neutral block.

Our theoretical predictions are well supported by extensive numerical calculations by density-matrix renormalization techniques. First, we have determined the average shapes of polymer molecules. Our results indicate that at large fields the polymer chains can be found mainly in a few trap configurations, in excellent agreement with our predictions. Furthermore, the dependence of the drift velocity on external field has been investigated. It confirms that the drift velocity of polyelectrolytes and polyampholytes decays exponentially in the large field limit, and for polyelectrolytes the decay exponent depends on a polymer length. In contrast to fully charged polymers, for chains consisting of neutral and charged blocks the drift velocity approaches the constant values determined by the size of the neutral blocks.

The importance of our analytical and numerical results on the behavior of repton model at large fields for real systems, e.g., gel electrophoresis, is unclear. $\stackrel{4}{*}$ The repton model does not take into account the creation of hernias and the transfer of tension forces, which are very important for the dynamics of polyelectrolytes at large fields. However, one may naively expect that the creation of hernias is less important for PA and for the polymers with neutral and charged blocks. Despite its limited applicability to gel electrophoresis, our calculations provide exact asymptotic results for several classes of asymmetric simple exclusion processes with two types of of oppositely moving particles, ${ }^{29}$ where the number of exact results is very limited.

\section{Acknowledgments}

ABK acknowledges the Donors of the American Chemical Society Petroleum Research Fund (Grant No. 37867-G6) for support of this research. ABK also acknowledges the financial support of the Camille and Henry Dreyfus New Faculty Awards Program (under Grant 
NF-00-056), the Welch Foundation (under grant No. C-1559) and Center for Biological and Environmental Nanotechnology at Rice University. This work has been also supported by the Polish Science Committee (KBN) under grant in years 2003-2005. We also thank M.E. Fisher and B. Widom for critical discussions, and J.M.J. van Leeuwen for careful reading of the manuscript.

1 P.G. de Gennes, Scaling Concepts in Polymer Physics, Cornell University Press, Ithaca, USA 1971.

2 M. Rubinstein, Phys. Rev. Lett. 59, 1946 (1987).

3 T.A.J. Duke, Phys. Rev. Lett. 62, 2877 (1989); T.A.J. Duke, J.Chem. Phys. 93, 9049 (1990); T.A.J. Duke, J.Chem. Phys. 93, 9055 (1990).

4 J.-L. Viovy, Rev. Mod. Phys. 72, 813 (2000).

5 B. Widom, J.-L. Viovy, and A.D. Defontaines, J. Phys. I 1, 1759 (1991).

6 J.M.J. van Leeuwen, J.Phys. I 1, 1675 (1991).

7 J.M.J. van Leeuwen and A. Kooiman, Physica A 184, 79 (1992).

8 A. Kooiman and J.M.J. van Leeuwen, Physica A 194, 163 (1993).

9 A. Kooiman and J.M.J. van Leeuwen, J. Chem. Phys. 99, 2247 (1993).

10 M. Prähofer and H. Spohn, Physica A 233, 191 (1996).

11 A.B. Kolomeisky and B. Widom, Physica A 229, 53 (1996).

12 R.D. Willmann, J. Chem. Phys. 116, 2688 (2002).

13 G.T. Barkema, J.F. Marko, and B. Widom, Phys. Rev. E 49, 5303 (1994).

14 M.E.J. Newman and G.T. Barkema, Phys. Rev. E 56, 3468 (1997).

15 E. Carlon, A. Drzewiński, and J.M.J. van Leeuwen, Phys. Rev. E 64, 010801(R) (2001).

16 E. Carlon, A. Drzewiński, and J.M.J. van Leeuwen, J. Chem. Phys. 117, 2435 (2002).

17 G.T. Barkema and G.M Schütz, Europhys. Lett. 35, 139 (1996).

18 G.M. Schütz, in Phase Transitions and Critical Phenomena, Eds. C. Domb and J. Lebowitz, Academic, London, 2000. Vol. 19.

19 S. R. White, Phys. Rev. Lett. 69, 2863 (1992); S. R. White, Phys. Rev. B 48, 10345 (1993).

20 T. Nishino, J. Phys. Soc. Jpn. 64, 3598 (1995); E. Carlon and A. Drzewiński, Phys. Rev. Lett. 79, 1591 (1997); A. Drzewiński, A. Maciołek, and R. Evans, Phys. Rev. Lett. 85, 3079 (2000). 
21 M. Kaulke and I. Peschel, Eur. Phys. J. B 5, 727 (1998); E. Carlon, M. Henkel and U. Schollwöck, Eur. Phys. J. B 12, 99 (1999).

22 Lectures Notes in Physics. V.528 ed. by I. Peschel, X. Wang, M.Kaulke and K. Hallberg (Springer, Berlin 1999).

23 G. H. Golub and C. F. Van Loan, Matrix Computations (Baltimore, 1996).

24 S.B. Smith, P.K. Aldridge and J.B. Callis, Science 243, 203 (1989).

25 L. Ulanovsky, G. Drouin and W. Gilbert, Nature 343, 190 (1990).

26 A. Kooiman, PhD Thesis, University of Leiden, 1993.

27 A. van Heukelum and H.R.W. Beljaars, J. Chem. Phys. 113, 3909 (2000).

28 A. Drzewiński, E. Carlon and J.M.J. van Leeuwen, arXiv:cond-mat/03065252, accepted to Phys. Rev. E.

29 G.M. Schütz, J. Phys. A: Math. Gen. 36, R339 (2003). 


\section{Figure Captions:}

Fig. 1 a) Two-dimensional representation of DNA molecule in a gel. Crosses represent gel fibers around which the chain is entangled. Dots mark the midpoints of segments;

b) Repton representation of DNA in a gel. A single spatial coordinate $x$ is in the direction of an externally applied electric field $\varepsilon$. The arrows represent allowed moves.

Fig. 2 An illustration of the correspondence between a configuration of the repton model, and a configuration in the asymmetric simple exclusion model with two species of particles.

Fig. 3 Diagrammatic picture for the polymer of size $N=3$. Arrows between boxes indicate allowed transitions between configurations.

Fig. 4 Trap configurations for polyelectrolyte chains of size $N=3,4$ and 5 .

Fig. 5 a) Part of the diagram for $N=5$ chain near the non-real trap configuration.

b) Part of the diagram for $N=5$ chain near the real trap configuration.

Fig. 6 The average shape of a polyelectrolyte chain of size $N=9$ for different fields.

Fig. 7 Plot of the logarithm of a drift velocity as a function of an electric field for chains with different length $N$. Inset: the plot $v$ vs. $\varepsilon$ for the same lengths.

Fig. 8 The average shape of an alternating chain of size $N=11$.

Fig. 9 Plot of the logarithm of a drift velocity as a function of an electric field for alternating chains with different length $N$. The dependence of a drift velocity for $N=3$ follows Eq.(5). Inset: the plot of $v$ vs. $\varepsilon$ for the same lengths. 
Fig. 10 The average shape of a chain of size $N=13$ where all reptons are neutral except one (left) end repton.

Fig. 11 Plot of $v$ vs. $\varepsilon$ for various $N$. The dashed lines are limiting values of a drift velocity according to Eq.(18) for various $N$ when $k=1$.

Fig. 12 Plot of $v$ vs. $\varepsilon$ for various $N$. The dashed lines are limiting values of a drift velocity according to Eq.(18) for various $N$ when $k=2$. 


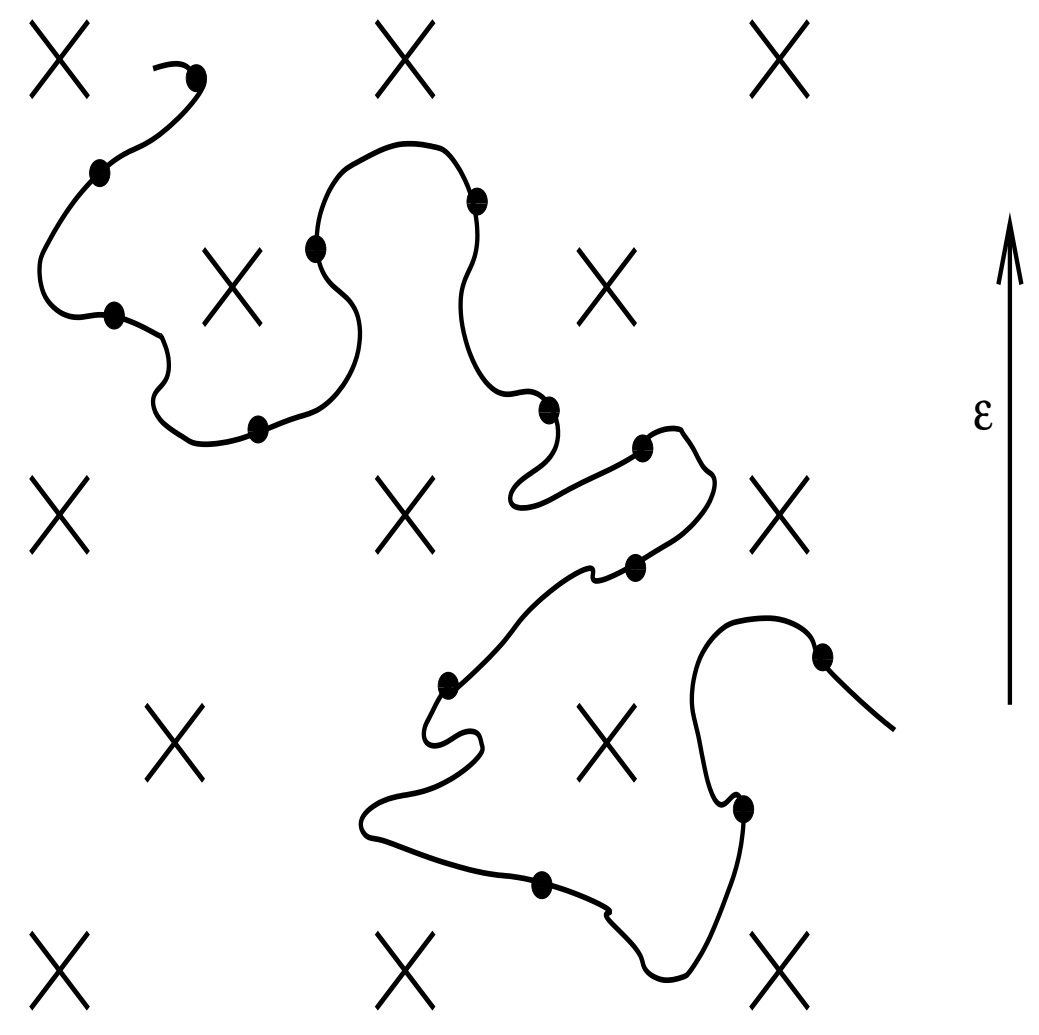

Fig.1a 


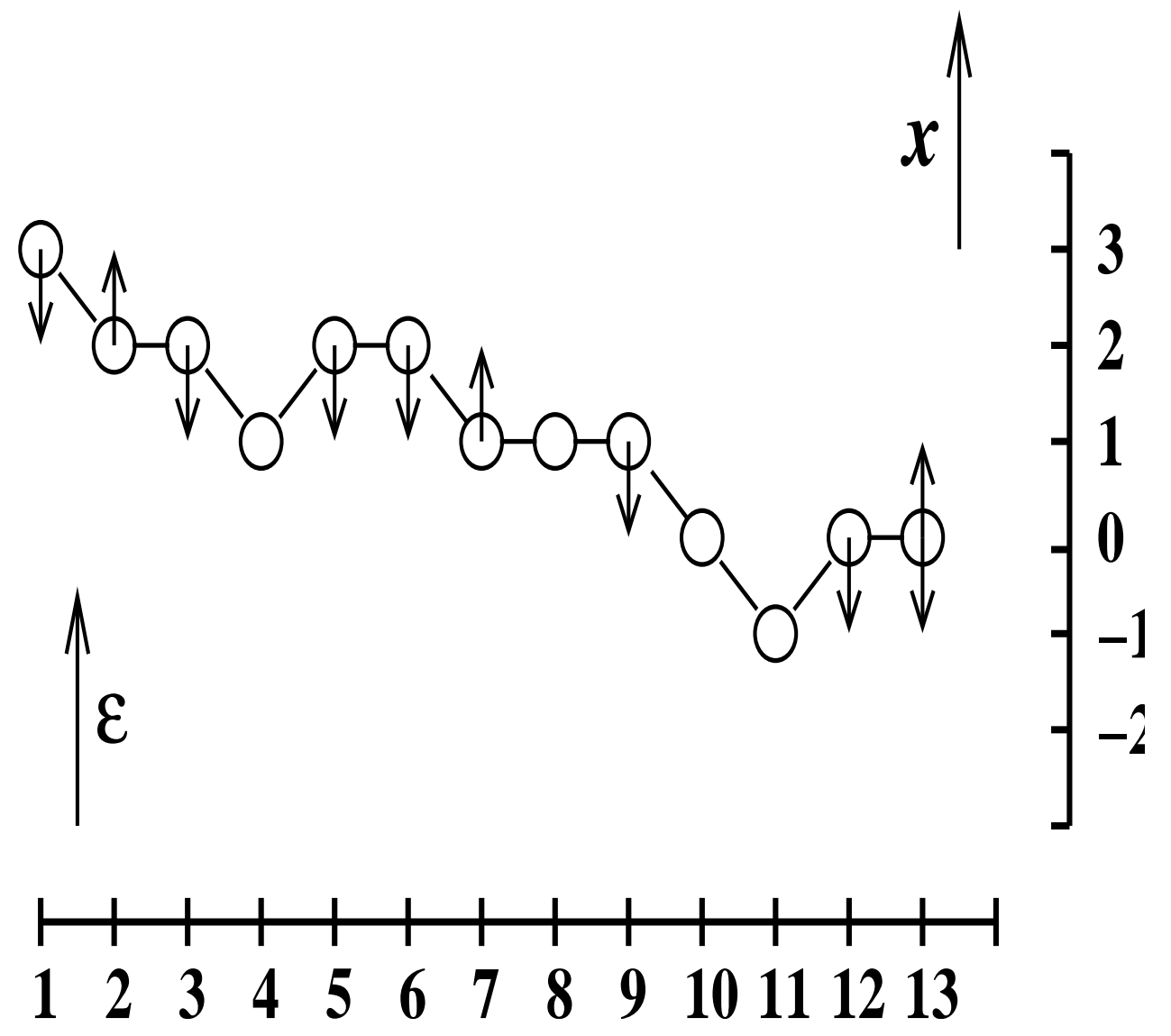

Fig.1b 


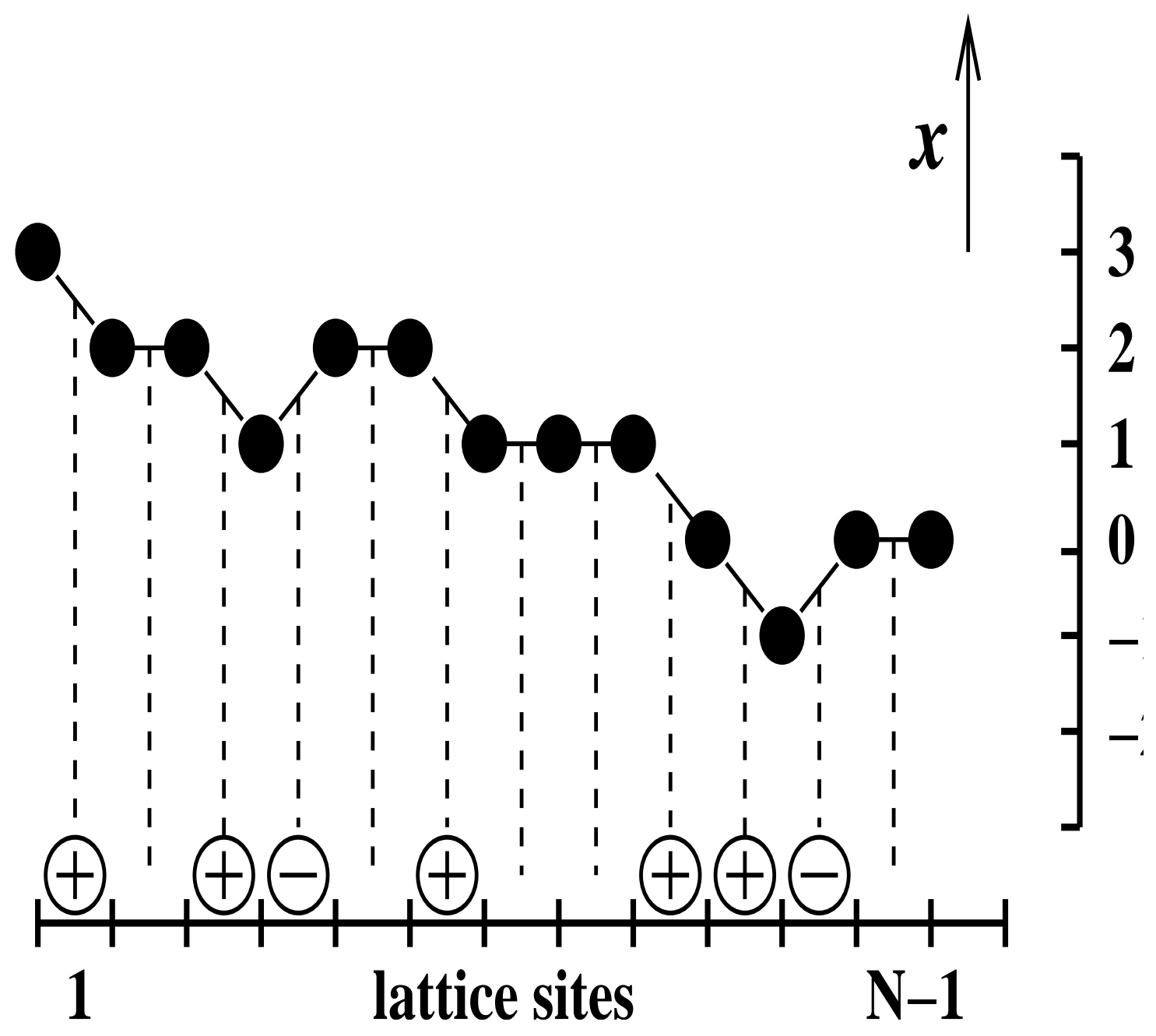

Fig.2 


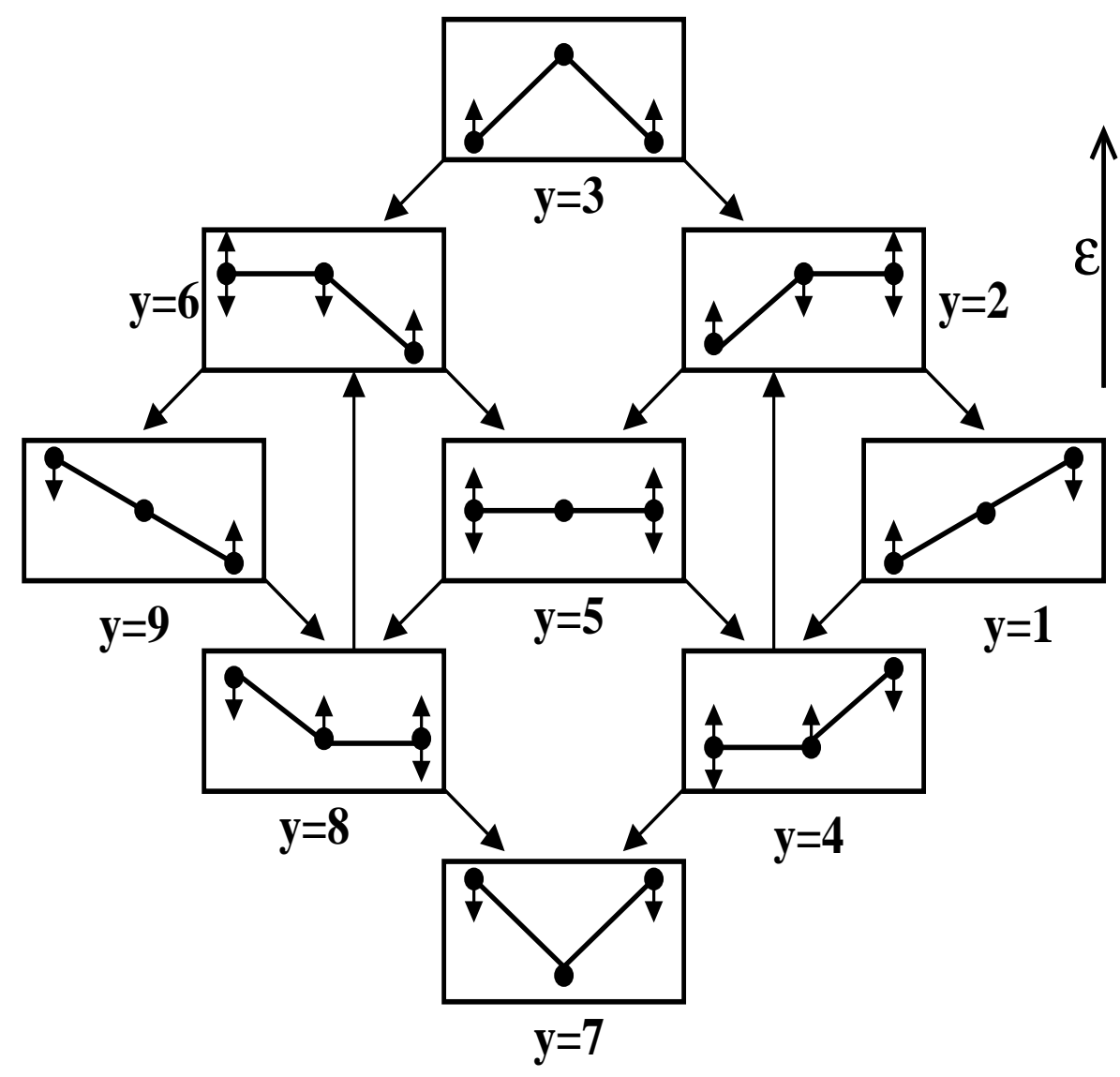

Fig.3 


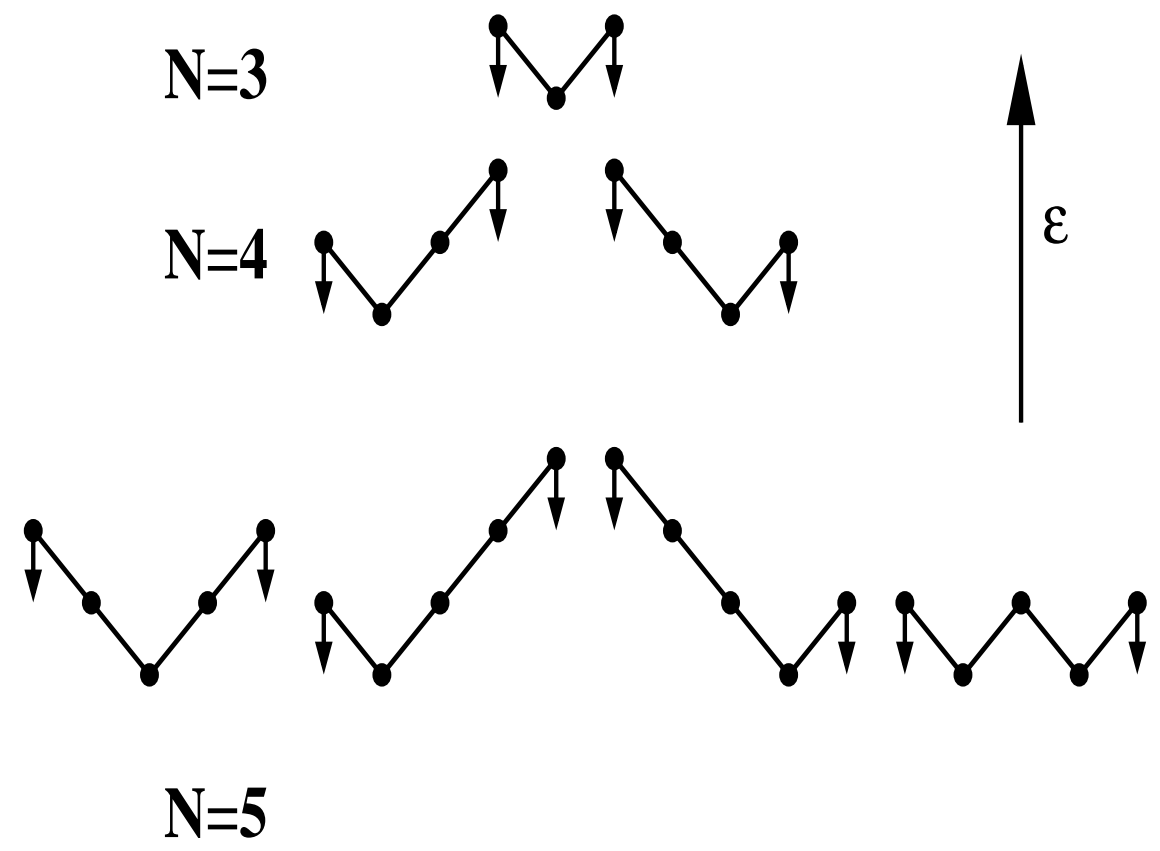

Fig.4 


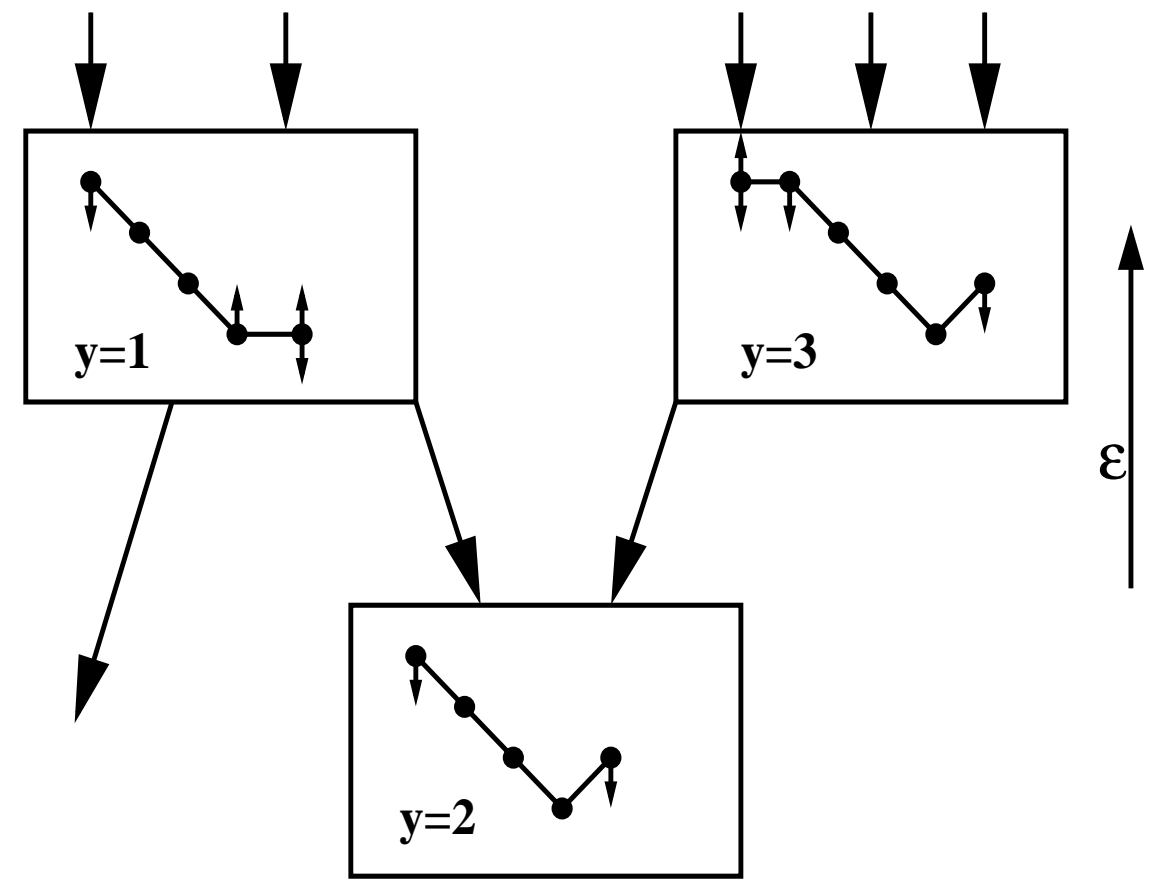

Fig.5A 


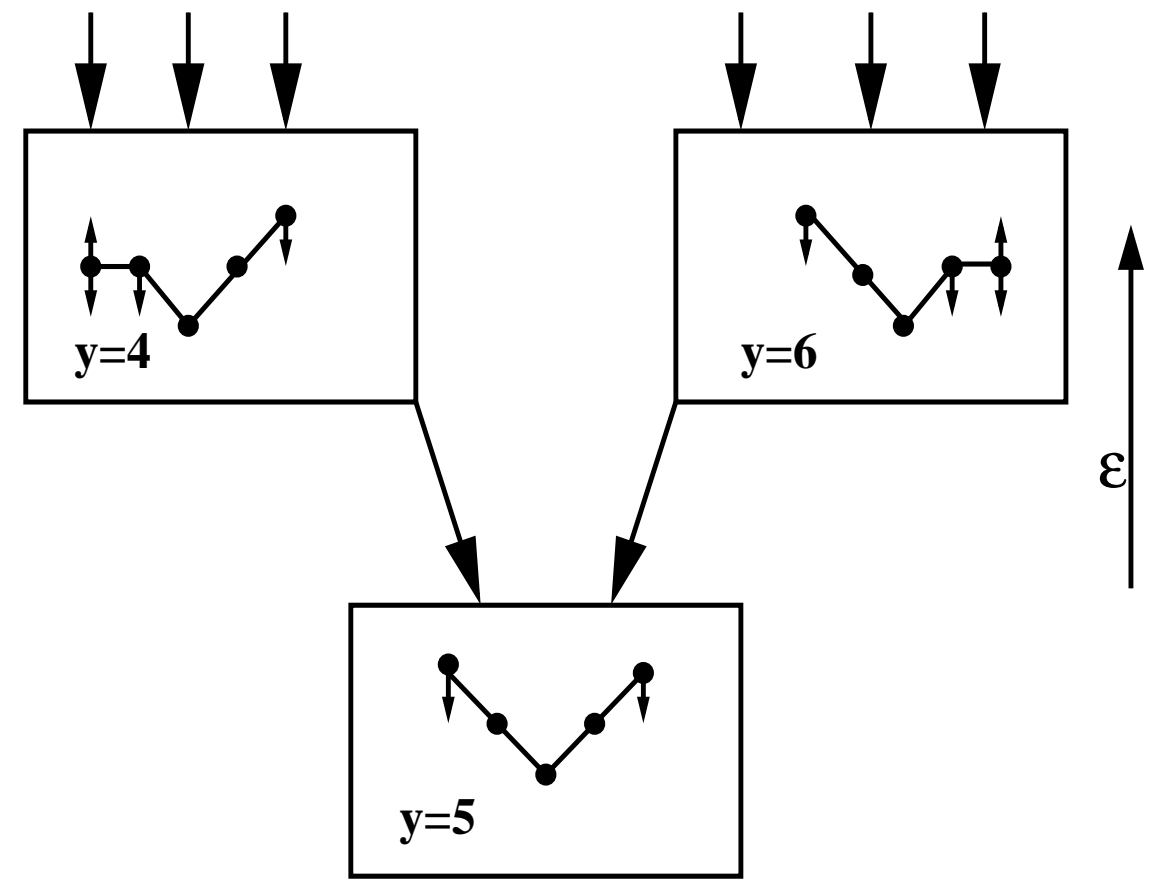

Fig.5B 


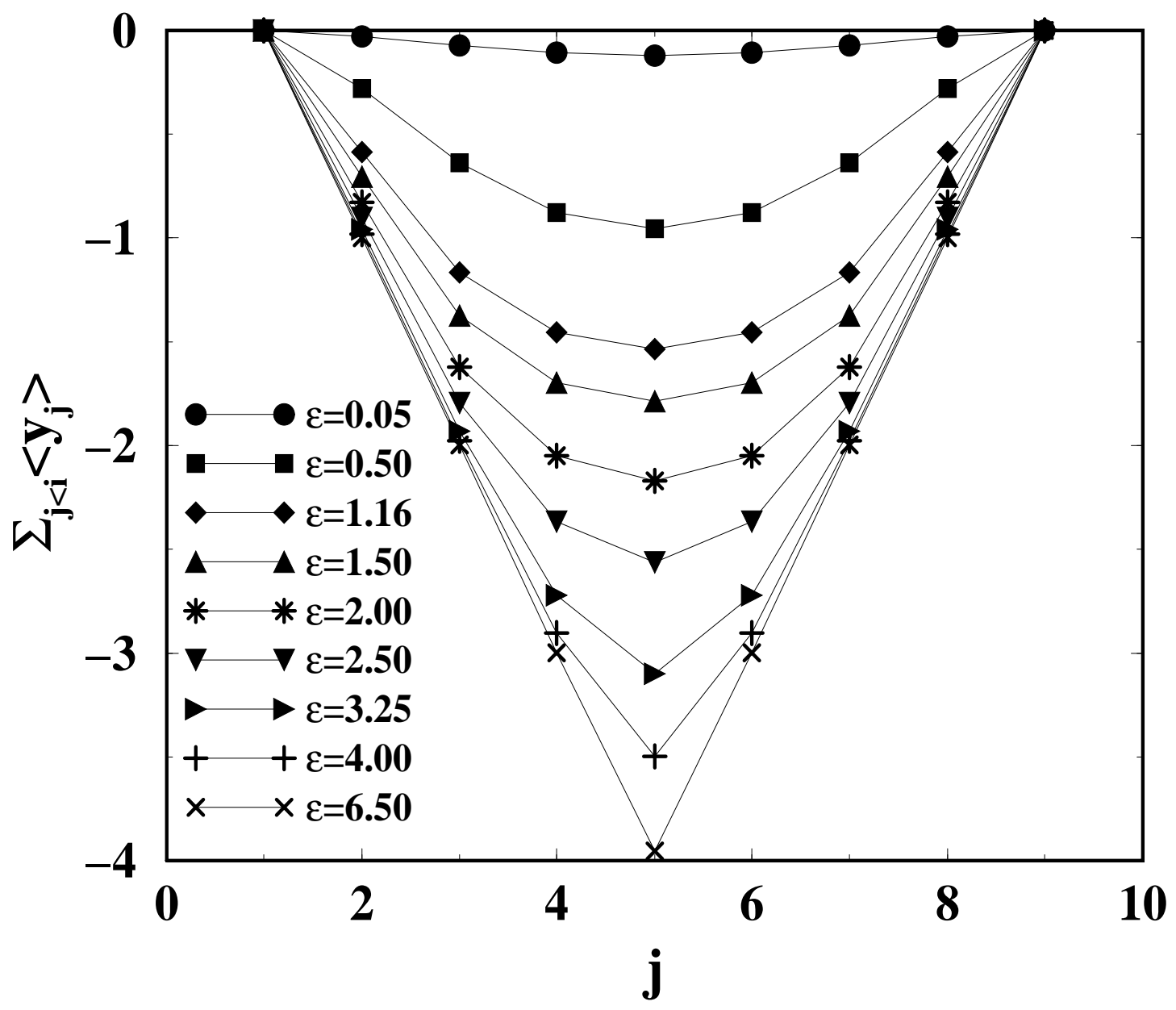

Fig.6 


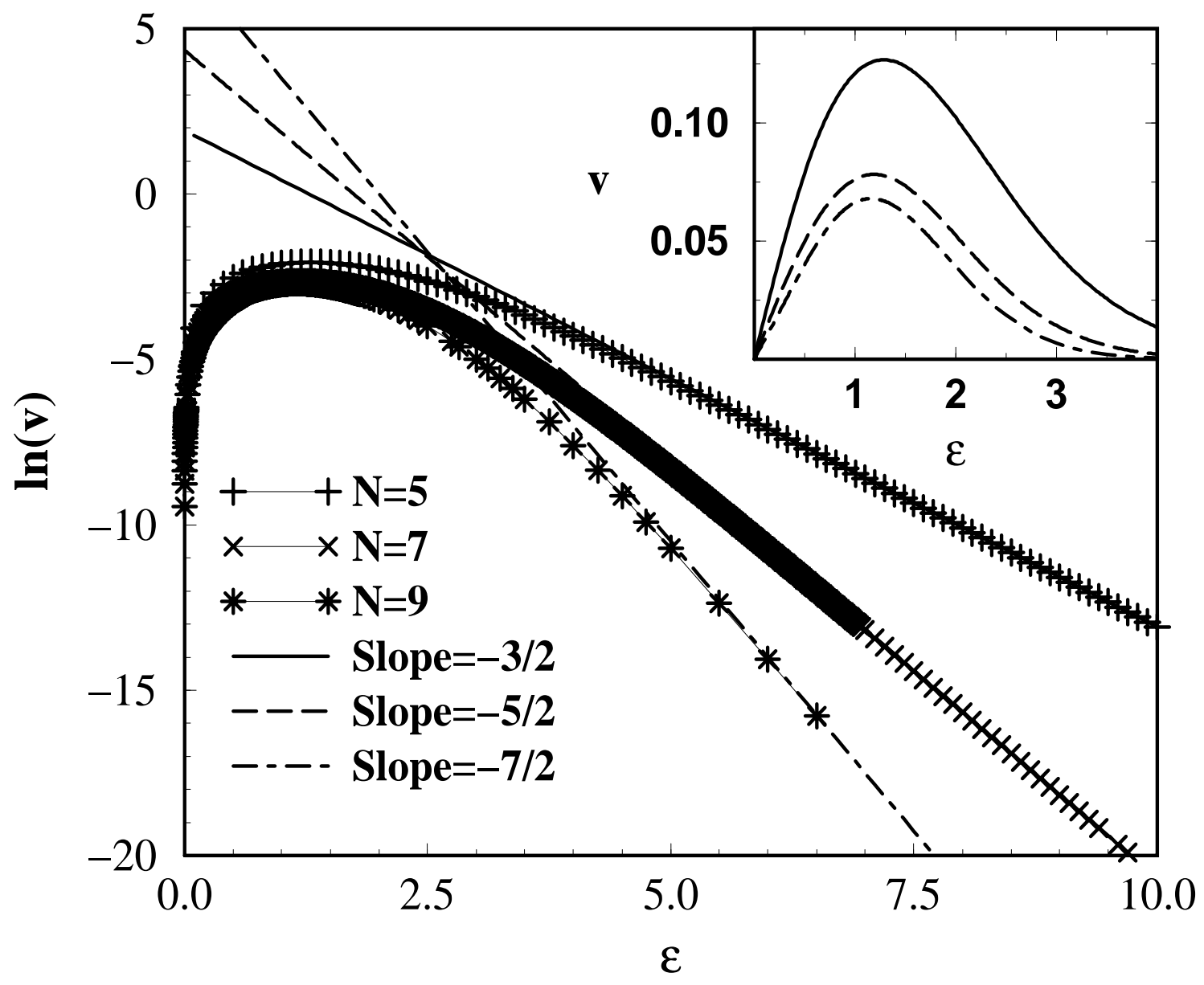

Fig.7 


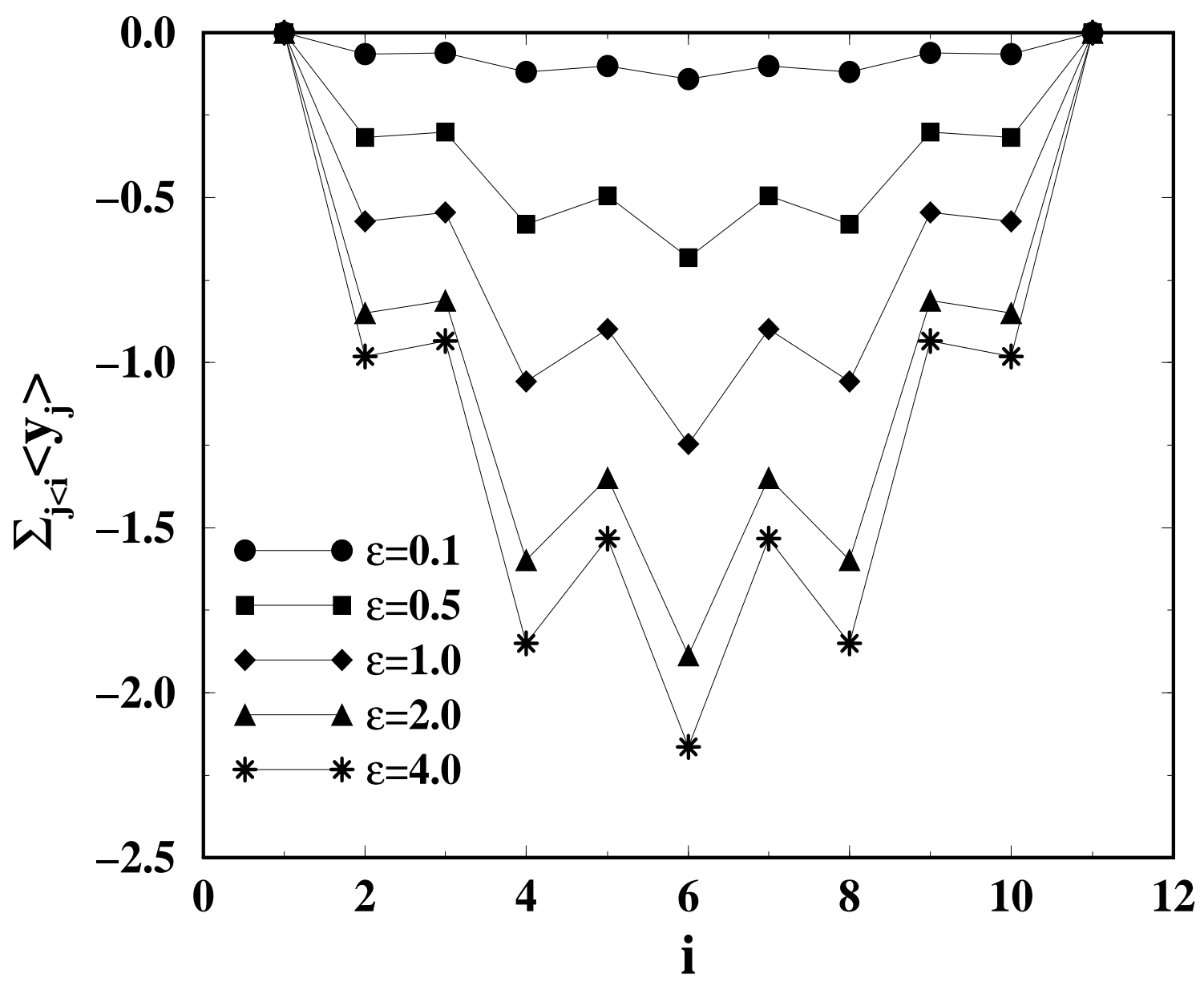

Fig.8 


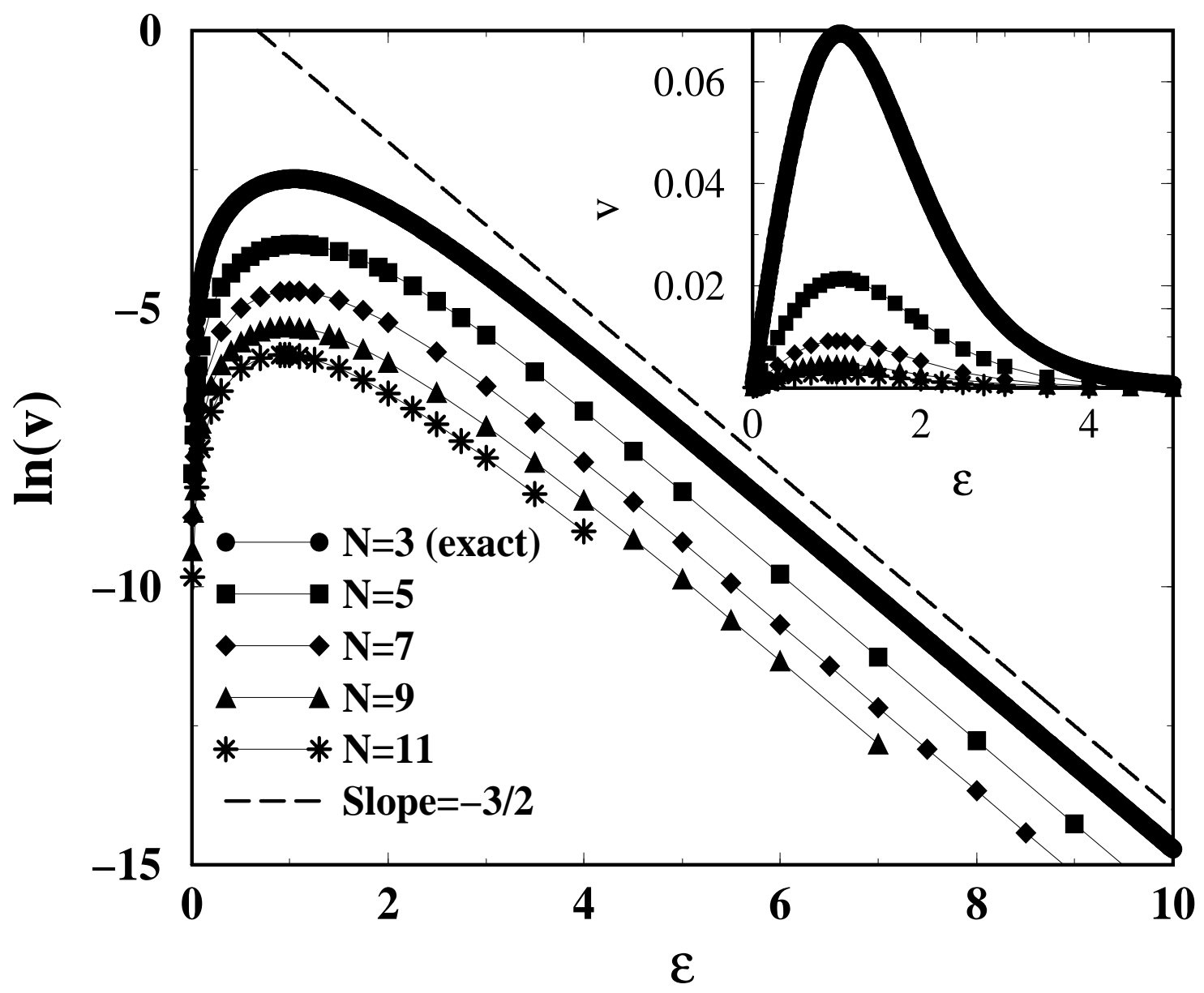

Fig.9 


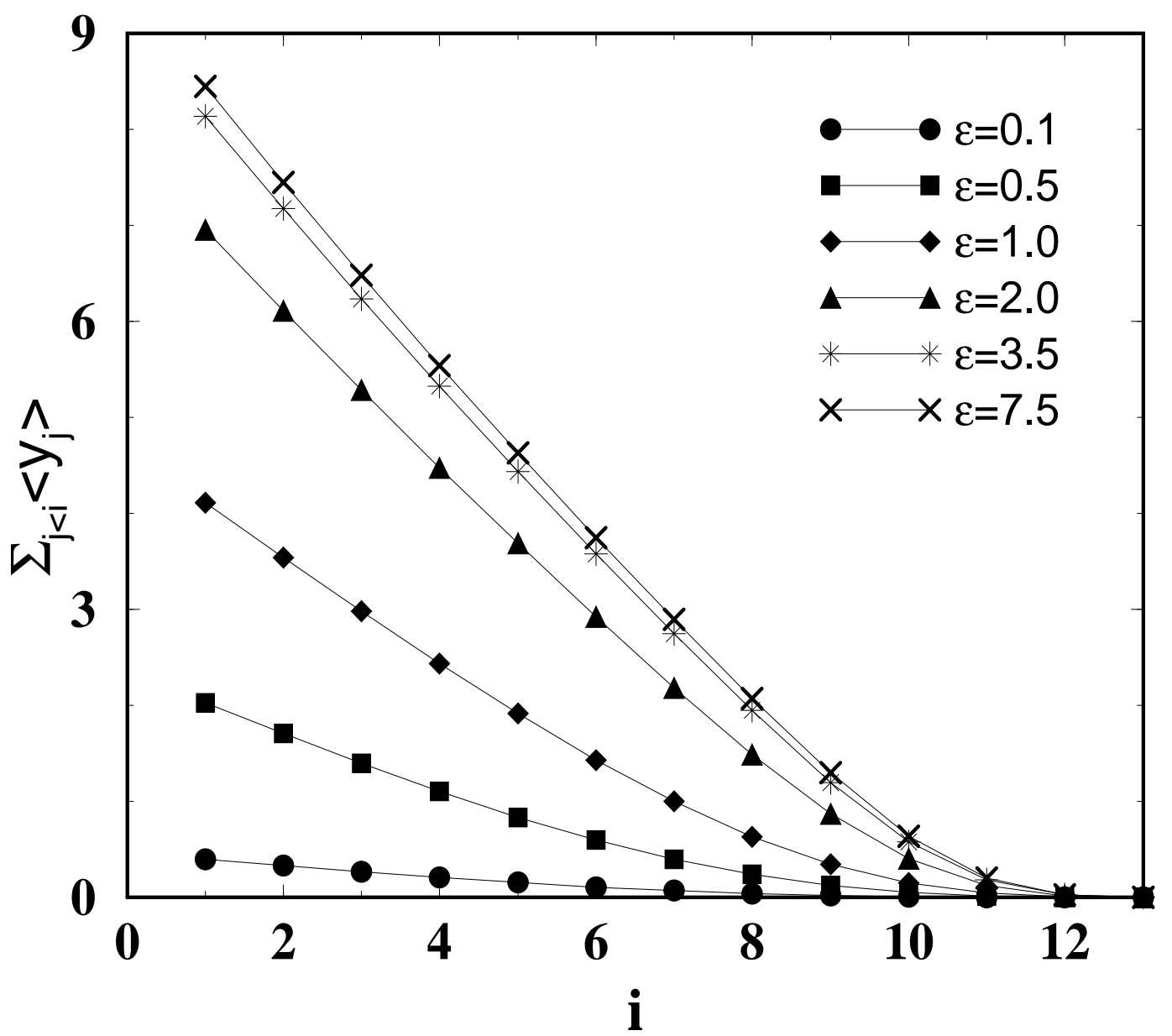

Fig.10 


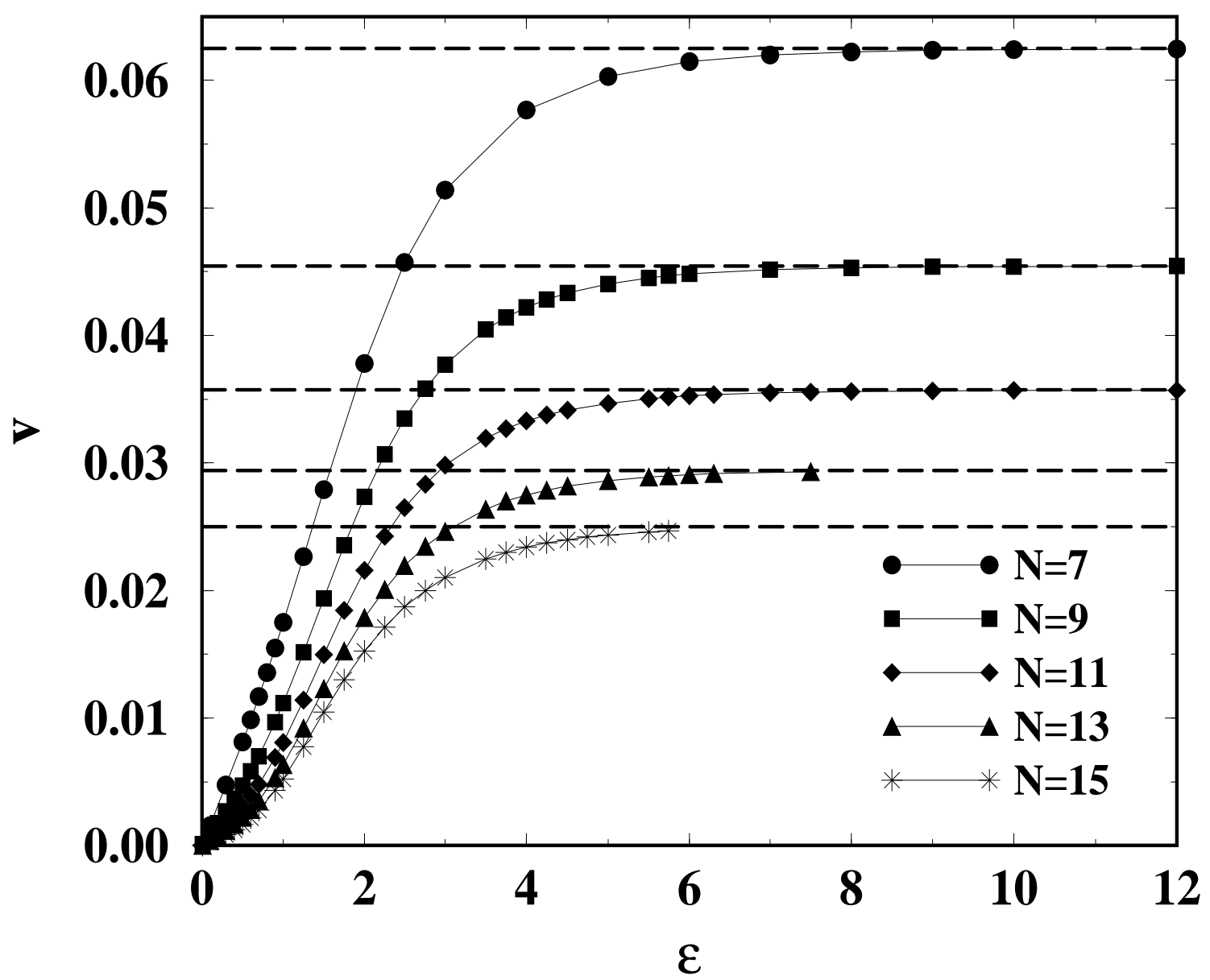

Fig.11 


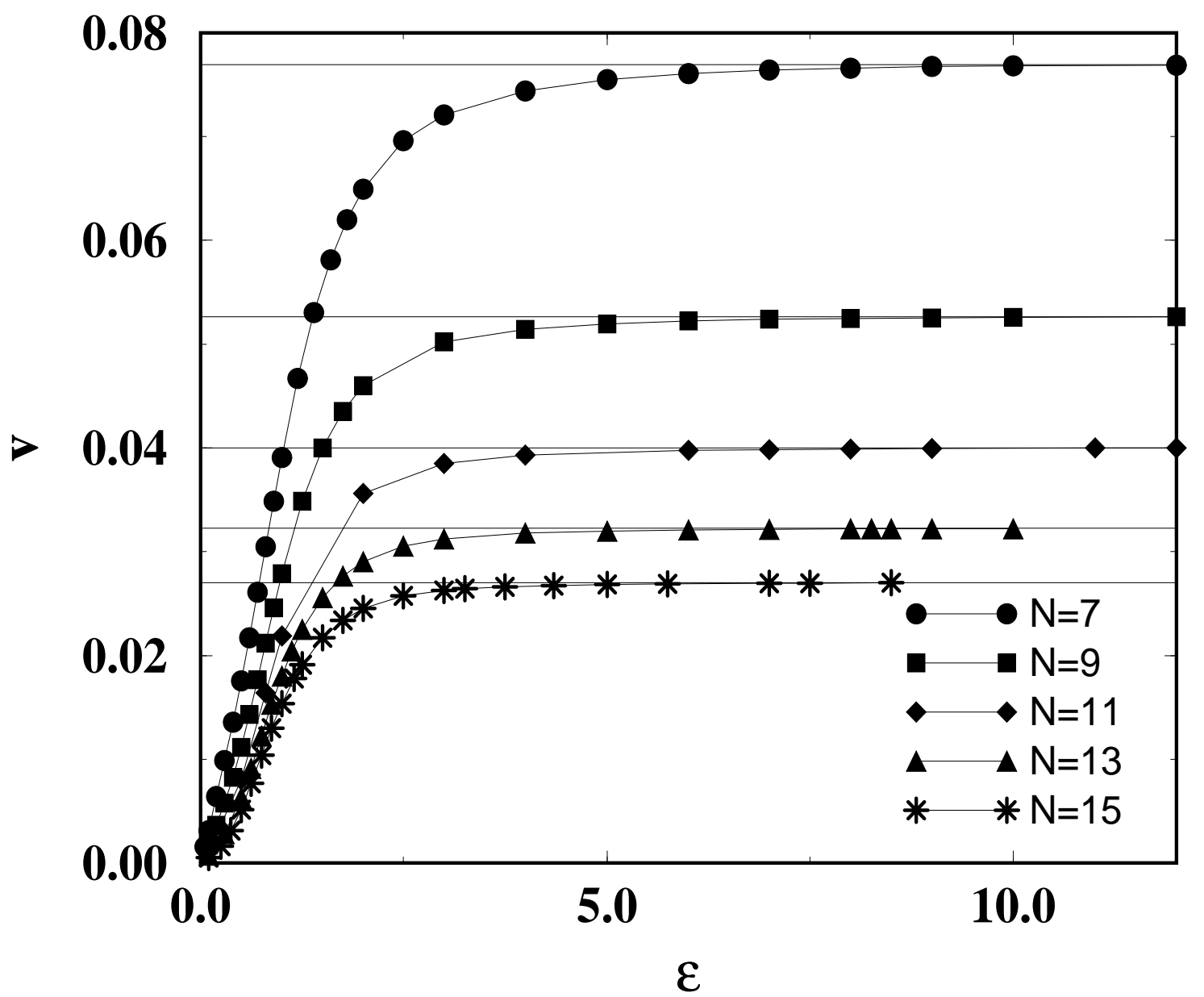

Fig.12 